\title{
Uso del enfoque cLIL como estrategia de internacionalización del currículo en una institución de educación superior colombiana
}

CLIL Approach Used as a Curriculum Internationalization

Strategy in a Colombian Higher Education Institution

Uso do enfoque AICL como estratégia de internacionalização

do currículo numa instituição de educação superior colombiana

Sara Isabel MONTOYA

Fundación Universitaria de San Gil - Unisangil, San Gil, Colombia

Corresponding author: smontoya@unisangil.edu.co

Orcid: http://orcid.org/00oo-0002-6644-307X

Carolina SALAMANCA

Fundación Universitaria de San Gil - Unisangil, San Gil, Colombia Corresponding author: csalamancaı@unisangil.edu.co

Orcid: http://orcid.org/0000-0002-4344-0538

Received: 2016-12-19

Accepted by peers: 2017-05-09
Send to for peer review: 2017-02-14

Approved: 2017-05-19

To reference this article in APA style / Para citar este artículo en APA / Para citar este artigo Montoya, S. I. \& Salamanca, C. (2017). Uso del enfoque AICLE/CLIL como estrategia de internacionalización del currículo en una institución de educación superior colombiana. Latin American Journal of Content and Language Integrated Learning, 10(1), 105-131. doi: 10.5294/laclil.2017.10.1.5 
RESUMEN. Este proyecto surge de la necesidad de responder a las políticas de "internacionalización en casa" de una institución de educación superior (IEs) coherente con las metas propuestas por el Ministerio de Educación Nacional en cuanto al desarrollo de competencias comunicativas de la lengua extranjera inglés para tales instituciones en el país. El diseño de la investigación tuvo una metodología descriptiva usada en dos procesos paralelos: la formación docente en las competencias comunicativas del inglés a partir de un diagnóstico y la capacitación docente en el Aprendizaje Integrado de Contenidos y Lenguas Extranjeras (AICLE; en inglés Content and Language Integrated Learning, CLIL) para el diseño y aplicación de actividades de contenido a través del trabajo colectivo de un grupo de profesores voluntarios de la IEs. Los resultados fueron positivos indicando que al hacer uso de recursos didácticos interesantes y motivadores, los estudiantes y docentes reconocen las ventajas pedagógicas para el aprendizaje de contenidos y de la lengua extranjera inglés, y a la vez desean continuar y ampliar el uso de las actividades de AICLE. Esto permitió ampliar el proyecto a un nivel institucional, socializar las experiencias y dar lugar a una segunda fase investigativa que valide la información.

Palabras clave: CLIL; desarrollo de competencias; eficiencia de la educación; estrategia de enseñanza; formación profesional superior.

ABSTRACT. This project emerged from the necessity of responding to the policies for "home internationalization" of a higher education institution in coherence with the aims proposed by the National Ministry of Education in relation to the development of communicative competences of English as a foreign language regarding this kind of institutions. The research had a descriptive design methodology used in a two parallel processes: a teaching training in the communicative competences of English starting from a diagnosis, and training in the CLIL approach for the design of content activities was implemented through the collective work of a team of volunteer teachers from the institution. After the activities were applied, the results were positive; indicating that making use of interesting and motivational didactic resources, students and educators recognized the pedagogical advantages of the approach for the learning of content simultaneously reinforcing communicative competences in English as a foreign language, and at the same time sought to continue and increase the use of CLIL activities. This allowed expanding the scope to an institutional level, socializing the experiences, and giving place to a second phase that can validate the information.

Keywords: CLIL; development of competences; effectiveness of education; higher vocational training; teaching strategy.

RESUMO. Este projeto surge da necessidade de responder às políticas de "internacionalização em casa" de uma Instituição de Educação Superior (IEs) coerente com as metas propostas pelo Ministério da Educação Nacional no que se refere ao desenvolvimento de competências comunicativas da língua estrangeira inglês para essas instituições no país. A pesquisa teve uma metodologia descritiva usada em dois processos paralelos: a formação docente nas competências comunicativas do inglês a partir de um diagnóstico e a capacitação docente na Aprendizagem Integrada de Conteúdos e Línguas Estrangeiras (AICL; em inglês Content and Language Integrated Learning, CLIL) para a elaboração e a aplicação de atividades de conteúdo por meio do trabalho coletivo de um grupo de professores voluntários da IEs. Os resultados foram positivos e indicaram que, ao fazer uso de recursos didáticos relevantes e motivadores, os estudantes e os docentes reconhecem as vantagens pedagógicas para a aprendizagem de conteúdos e da língua estrangeira inglês, e ao mesmo tempo desejam continuar e ampliar o uso das atividades de AIcL. Isso permitiu estender o projeto a um nível institucional, socializar as experiências e realizar uma segunda fase de pesquisa que possa validar a informação.

Palavras-chave: AICL; desenvolvimento de competências; eficiência da educação; estratégia de ensino; formação profissional superior. 


\section{Introducción}

El problema de investigación surge desde la necesidad de responder al proceso de globalización actual, en donde la aproximación y apropiación del conocimiento dista de la forma habitual como se desarrolló durante décadas, el cual exige, como lo presenta la Organización de las Naciones Unidas para la Educación, la Ciencia y la Cultura dentro de sus objetivos educativos: "Responding to contemporary global challenges through education"1 (Unesco, 20I I), para ello se espera que los ciudadanos, entre otras competencias, puedan intercambiar información haciendo uso de la lengua extranjera inglés (Chomsky, I965).

Tal responsabilidad recae, a través del Ministerio de Educación Nacional (MEN) sobre las instituciones de educación del país y la institución, con el fin de asumir este reto, presenta unas directrices muy claras dentro de las estrategias de internacionalización para todos sus programas académicos respecto a la lengua extranjera inglés y su uso en el desarrollo de las clases y aplicación en todas las asignaturas; por tanto, se hace necesario implementar estrategias asertivas que contribuyan al mejoramiento de las competencias en inglés de los estudiantes desde la formación docente.

En el país, durante las últimas dos décadas, se viene notando un alto grado de interés en torno al desarrollo de competencias comunicativas en la lengua extranjera inglés de los colombianos, razón por la cual el MEN ha puesto en marcha proyectos como el Programa Nacional de Bilingüismo, el Programa de Fortalecimiento al Desarrollo de Competencias en Lenguas Extranjeras y el Programa Nacional de Inglés 2015-2025 en donde se pudo evidenciar, a través de un diagnóstico realizado, que más del 50\% de los estudiantes avanzaron un nivel de inglés o más durante sus estudios de educación superior (Ministerio de Educación Nacional, 20I4).

Con el fin de asumir el desafío del bilingüismo dentro de los proyectos lanzados por el MEN y bajo la estrategia de "internacionalización

1 "Respondiendo a los desafíos globales contemporáneos a través de la educación”. Extraído del documento Everyone has the right education de la Unesco. 
en casa"2, se presenta esta propuesta basada en un plan de trabajo para la formación docente en el enfoque cLIL, así como en las competencias comunicativas del inglés. Esta iniciativa además está orientada en mejorar la calidad educativa de los estudiantes y está enfocada a través de proyectos formativos que buscan alcanzar los objetivos propuestos por el MEN los cuales permiten identificar y comprobar estrategias comunes en las iEs con avances significativos en:

a. Docentes. La formación docente se enfoca en robustecer las capacidades en pedagogía, se cuenta con la presencia de docentes nativos o con estudios en el exterior.

b. Modelo Pedagógico. La existencia de un modelo curricular alineado con el Marco Común Europeo, se oferta la enseñanza del inglés de forma flexible y adaptable a las necesidades, facultades específicas que cuentan con contenidos de área obligatorios en inglés y la disponibilidad de materias electivas y actividades extracurriculares.

c. Cultura de evaluación y desempeño. Se diagnostica el nivel de inglés de los estudiantes al ingreso y a su salida, y se establecen requisitos de grado vinculados al aprendizaje.

d. Infraestructura de apoyo. Se garantizan grupos pequeños con acceso a infraestructura tecnológica de punta y acceso a software educativo de apoyo.

A partir de dichas estrategias el proyecto tiene como objetivo identificar la efectividad del uso del enfoque cLIL como estrategia de internacionalización del currículo a través de la formación docente en la enseñanza de contenidos integrando la lengua extranjera inglés y la valoración de los estudiantes de la iEs sobre el uso del mismo.

Para ello la IEs adopta dicho enfoque pedagógico en el cual se desarrollan procesos cognitivos usando una lengua diferente a la lengua materna (LI). CLIL "Hace referencia a cualquier contexto educativo en el que se utiliza una lengua extranjera L2 como medio de enseñanza y aprendizaje de contenidos no relacionados con la lengua en sí” (Suárez, 2005).

2 "internacionalización en casa" es el término usado por la iEs para referirse a los procesos de internacionalización del currículo. 
CLIL tiene como características el uso de diferentes tareas, el aprendizaje colaborativo, la integración a las diferentes formas de aprendizaje y las habilidades comunes para todas las lenguas. Tales características se enmarcan dentro de los principios de cLiL. De acuerdo con Do Coyle (2002) el primero de ellos es el Contenido, el cual coloca con éxito la adquisición de conocimientos, destrezas y comprensión inherentes a esa disciplina, en el corazón mismo del proceso de aprendizaje. El segundo principio, Comunicación, define al lenguaje como conducto para la comunicación y el aprendizaje. El tercer principio, Cognición, es que CLIL debe suponer un reto cognitivo para los alumnos: para que puedan desarrollar sus destrezas de pensamiento en conjunción con sus habilidades básicas de comunicación interpersonal y su competencia en el lenguaje cognitivo-académico. El cuarto principio, Cultura, comprende la multiculturalidad, ya que lengua, pensamiento y cultura se encuentran ligados.

\section{Método}

La investigación cuenta con un diseño cualitativo en cuanto a las observaciones y seguimiento registrados, tratándose de un estudio descriptivo, no enfocado en una presunta relación causa-efecto sino en la descripción de un caso específico sirviendo como inicio de posteriores investigaciones analíticas. Este tipo de investigación es considerada como Innovación Pedagógica por cuanto la metodología desarrollada en la misma no presenta antecedentes en el contexto universitario a nivel institucional con una población limitada en las competencias de la lengua extranjera inglés.

La investigación fue dirigida a 2IO docentes de las cuatro facultades de la IES y programas en extensión (i3 programas de pregrado y 5 de posgrado) vinculados a la misma bajo cualquiera de las modalidades contractuales, sin requisitos previos en las competencias comunicativas de la lengua extranjera inglés e interesados en hacer parte del proceso de formación docente. Para el estudio se determinó una muestra no probabilística intencional de: 
» 90 docentes a quienes se les evaluó el nivel de competencia en la lengua extranjera inglés y su interés por integrar éste en sus clases.

» II docentes voluntarios con interés en recibir capacitación en el enfoque CLIL.

\section{Procedimiento}

Para el desarrollo del proyecto se llevaron a cabo las siguientes etapas:

Estudio de las necesidades de la institución: para comenzar el proyecto, inicialmente se realizó el diseño de una encuesta virtual para obtener los resultados y estadísticas sobre las opiniones y aspectos motivacionales que los docentes tienen hacia el área de inglés y su implementación dentro de las clases académicas. Se realizó el pilotaje de dicha encuesta con docentes de diferentes programas de la institución y después se hizo una convocatoria interna por programas para aplicar la encuesta virtual junto con la prueba de clasificación de inglés con el fin de diagnosticar los niveles referidos dentro del Marco Común Europeo (MCE). Finalmente, se realizó el análisis de los resultados y consolidación de datos con el fin de determinar el estado inicial de la población beneficiaria de la investigación.

Formación en el enfoque cLIL y en las competencias comunicativas del inglés: En la primera etapa se realizó una convocatoria abierta a todos los docentes de la institución con el fin de consolidar un grupo de estudio de mínimo un docente por programa académico para participar en el proyecto. Después se instauraron encuentros generales y una metodología a seguir con los docentes inscritos y el acompañamiento de un docente asesor en CLIL, estableciendo:

a. Cada docente seleccionó el contenido y competencias sobre las cuales aplicó el enfoque cLIL en una las asignaturas que orienta.

b. El docente asesor en enfoque CLIL programó y realizó visitas a dichas clases seleccionadas por los docentes con el fin de analizar la metodología de enseñanza y conocer previamente el contenido.

c. El docente asesor realizó capacitaciones con el fin de instruir a los docentes participantes en el enfoque CLIL. 
d. Una vez capacitados, los docentes realizaron el diseño del instrumento planeador de clase $^{3}$ mediante un trabajo personalizado entre cada docente y el asesor en CLIL.

e. El docente construyó el material didáctico para la aplicación del planeador de clase.

Durante el proceso se realizó al mismo tiempo capacitaciones en inglés para mejorar el nivel de los docentes de la institución de acuerdo con los datos obtenidos en la prueba de clasificación.

Aplicación de la propuesta: Una vez finalizada la construcción del planeador de clase con el enfoque cLIL se hicieron las aplicaciones diseñadas por los profesores. Después del desarrollo de las actividades se evaluaron los resultados obtenidos para presentar los informes correspondientes a la comunidad educativa e incentivar a los docentes mediante estímulos autorizados por la institución.

Finalmente se implementaron los ajustes necesarios dentro del proyecto de acuerdo con los resultados para continuar promoviendo el desarrollo del mismo en los años posteriores e institucionalizarlo, realizando además la apertura de un espacio virtual en las principales redes sociales para promover y motivar a la comunidad a vincularse en el proyecto.

\section{Instrumentos y recolección de datos}

Para la recolección de la información producida en el desarrollo del proyecto se tienen en cuenta las siguientes técnicas de investigación y los respectivos instrumentos diseñados para tal fin:

La observación como método que permite "el uso sistemático de nuestros sentidos en la búsqueda de los datos que necesitamos para resolver un problema de investigación" (Sabino, I992) admitió conocer la realidad mediante la percepción directa de los objetos y fenómenos involucrados en el proyecto.

3 El planeador de clase o lesson planning como se denominó para los docentes, es un instrumento de planeación de clases adaptado de múltiples autores en el que se desarrollan los principios de CLIL. 
La encuesta de caracterización realizada a 90 docentes voluntarios de la institución, procesada con el programa spss versión 2.o, pretendió medir y determinar las opiniones y aspectos motivacionales que los docentes tienen hacia el área de inglés y su implementación en las diferentes clases de todos los programas académicos, para contrastarlos al finalizar la implementación del proyecto y así determinar su efectividad.

La prueba de clasificación en inglés la cual midió el nivel de competencia de la lengua extranjera inglés de los docentes de la institución, generada por el Instituto de idiomas de la IEs.

Los formatos de diseño de clases CLIL para los docentes voluntarios que planearon clases integrando el inglés en el 2015-2.

Las guías de trabajo en clase que dan cuenta del uso de las competencias comunicativas de los docentes capacitados.

La Encuesta de percepción de la metodología usada por parte de los docentes y los estudiantes involucrados, procesada con el programa SPSS versión 2.0.

\section{Resultados}

A continuación, se presentan los resultados de la investigación de acuerdo a los objetivos específicos planteados para el desarrollo de la misma.

El primer objetivo consistió en la determinación de algunos factores necesarios para el inicio del proceso investigativo como son la identificación de las competencias en lengua extranjera inglés y aspectos motivacionales para la integración de este idioma en las asignaturas de los docentes de la institución. Para responder este objetivo se usó como instrumento una encuesta de forma virtual antes de desarrollar la prueba de clasificación de inglés y fue resuelta por un total de 90 docentes de la institución en donde la mayor participación fue por parte de la Facultad de Ingeniería.

Algunas preguntas de la encuesta tuvieron como fin determinar la concepción que tienen los docentes acerca de su experiencia de aprendizaje de la lengua extranjera inglés: 3i\% de los docentes califica (en una escala de I a 5 siendo I el más bajo y 5 el más alto) como 3 la 
calidad del aprendizaje del idioma inglés que recibió en la institución que lo graduó como profesional. Esta respuesta permite apreciar la calidad en los métodos de enseñanza del inglés, lo cual fundamenta la necesidad de generar un proceso de capacitación pertinente y más motivadora para los docentes de la institución.

De acuerdo con la propia perspectiva de los docentes acerca de su nivel de competencia para cada una de las habilidades comunicativas del lenguaje (figura I), en las habilidades receptivas se encuentra que la mayoría califica su desempeño en escucha entre I y 2, en la habilidad de lectura se encuentra para la mayoría en 3 siendo ésta la habilidad en la que mejor se desempeñan los docentes según su apreciación. Para la habilidad de habla, la mayoría califica su desempeño en I así como en escritura.

Figura 1. ¿Cuál considera que es su desempeño en las habilidades comunicativas del idioma inglés?

\section{Porcentaje}

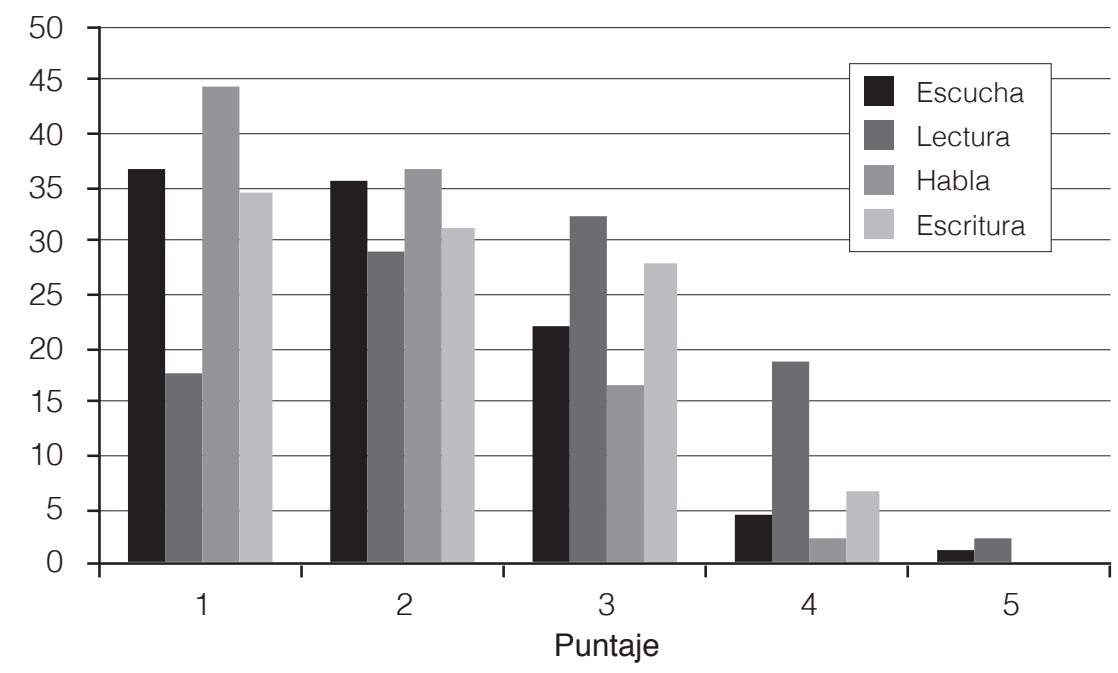

El siguiente grupo de resultados (para conocer las preguntas de la prueba de caracterización véase el apéndice i) determinó la motivación con la que cuentan los docentes para incursionar y aplicar la metodología CLIL en las asignaturas que orientan. 
Dentro de la concepción que cada docente tiene de la importancia sobre el dominio del inglés en su formación y desarrollo como profesional (figura 2), el $78 \%$ reconoce en alto grado la importancia del mismo. Este aspecto es fundamental en dos sentidos: por una parte, la importancia que le otorgan los docentes a capacitarse en la lengua extranjera inglés para avanzar académicamente en procesos de maestría y doctorado, y por otra parte, la necesidad de brindar a sus estudiantes mejores posibilidades de formación en este idioma.

El 94.5\% de los docentes opinan que es posible relacionar la enseñanza del inglés con otras asignaturas, pero al consultarles si consideran que están preparados y capacitados para orientar sus asignaturas usando el inglés, el 45.6\% considera que muy poco justificando así la asesoría que se encuentra en curso y la necesidad de la formación en el enfoque CLIL.

La mayoría de los docentes estimaron que se podría mejorar la motivación de sus clases si enseñan usando el inglés, también valoran que el aprendizaje de este idioma se puede mejorar si se trabaja a través del uso del idioma en sus asignaturas y viceversa. Este proceso es objeto de estudio del proyecto porque los docentes reconocen que adquirirán un mejor dominio en el idioma inglés si lo usan para enseñar los contenidos de sus asignaturas, y de esta forma se esperaba que los docentes que han hecho parte de la capacitación en CLIL mejoren su formación en inglés aprovechando los cursos propuestos, así como se esperaba que los docentes que se han capacitado en el idioma inglés deseen hacer parte de la capacitación CLIL.

Al consultar por el interés en la formación en el idioma inglés (figura 3) y su interés por integrarlo a las asignaturas (figura 4) se obtuvo una respuesta muy positiva en donde el porcentaje de docentes interesados fue muy alto.

Dado que se evidenció el interés por parte de los docentes, se procedió a determinar el nivel de inglés de cada uno de ellos con el fin de complementar su formación.

Prueba de clasificación de inglés. La prueba de clasificación de inglés aplicada a los 90 docentes voluntarios de todos los programas de la institución de forma virtual, es la misma realizada a los estudiantes de pregrado, consta de un cuestionario desde el nivel Starter hasta el nivel 6 en coherencia con los parámetros del Marco Común Europeo (MCE). 
Figura 2. Resultados de la prueba de caracterización.

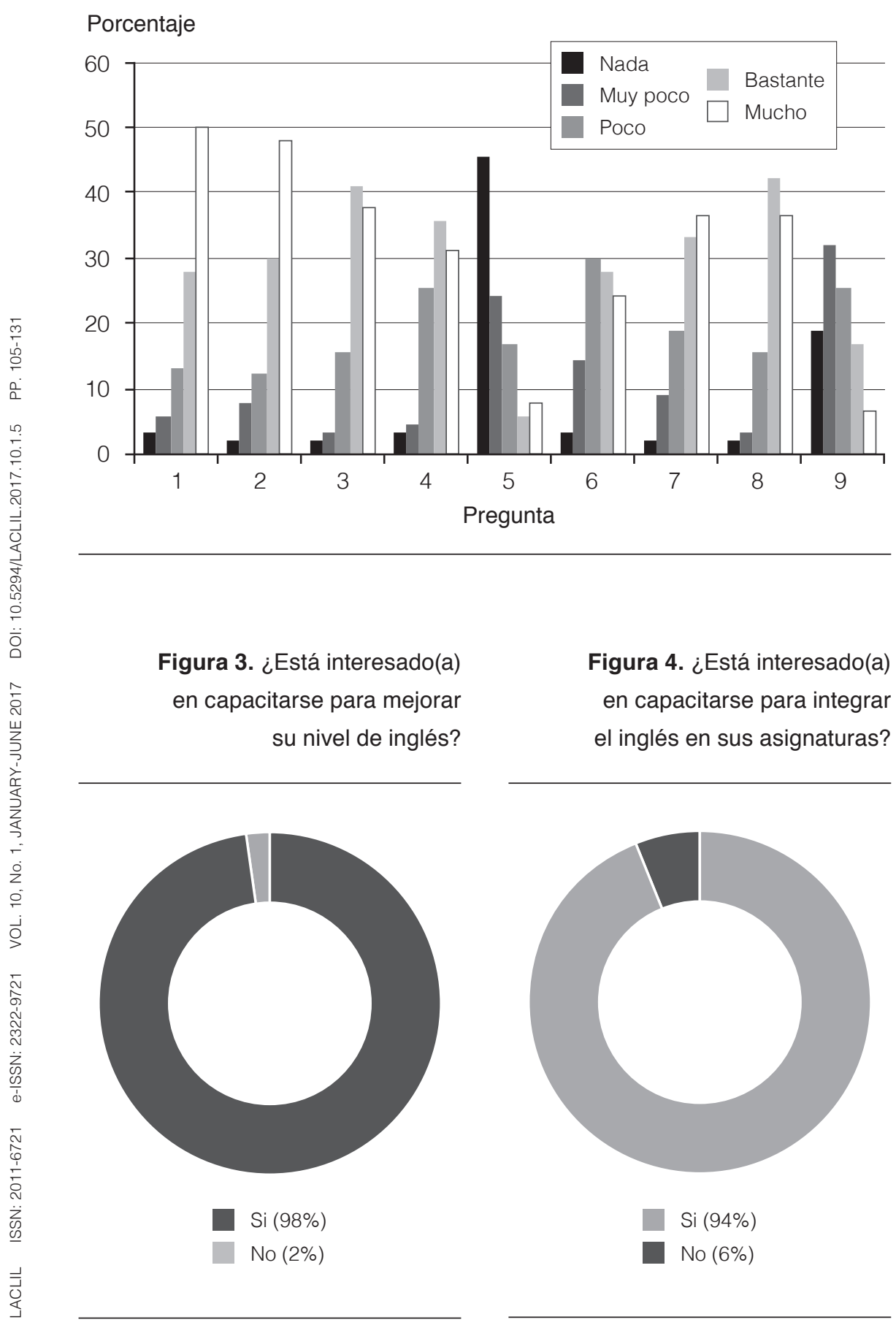


Los resultados obtenidos se resumen en la tabla I y muestran el nivel de clasificación en el que se encuentran los docentes interesados en la formación ofrecida por la institución a través del Instituto de Idiomas:

Tabla 1. Cantidad de docentes de acuerdo a la prueba de clasificación

\begin{tabular}{cccccccc}
\hline Nivel & Starter & Nivel 1 & Nivel 2 & Nivel 3 & Nivel 4 & Nivel 5 & Nivel 6 \\
\hline Docentes & 60 & 5 & 10 & 2 & 6 & 3 & 4 \\
\hline Porcentaje & $66,67 \%$ & $5,55 \%$ & $11,11 \%$ & $2,22 \%$ & $6,66 \%$ & $3,33 \%$ & $4,44 \%$ \\
\hline
\end{tabular}

En coherencia con estos resultados, donde aproximadamente el $67 \%$ de los docentes no alcanzaron el nivel AI (MCE) se lleva a cabo el desarrollo del segundo objetivo específico de la investigación que consistió en desarrollar una metodología para el fortalecimiento de las habilidades comunicativas en los docentes, con el fin de fortalecer las bases de la lengua extranjera necesarias para aplicar actividades con el enfoque cliL. Para ello se realizó una capacitación en el idioma inglés a los docentes de la institución. Desde los resultados obtenidos se ofertaron tres cursos para nivel introductorio, un curso para nivel 2 y uno para un nivel avanzado. Los docentes situados entre los niveles 3, 4 y 5 seleccionaron alguna de las siguientes opciones: retomar el aprendizaje desde el nivel 2, participar de las clases de inglés con los estudiantes de pregrado, o participar del nivel avanzado.

De los 90 docentes que presentaron la prueba, 69 se inscribieron en los cursos y terminaron participando 49, lo que indica una asistencia del 7I\% dando un balance positivo. Se encontró que el 68\% de los inscritos en los cursos habían realizado la prueba de clasificación, los demás lo hicieron antes de comenzar con el fin de permitir evidenciar los avances producidos en la formación en esta lengua extranjera.

Mientras que los docentes se formaban en el idioma inglés a través de los cursos, y a partir de los resultados del estudio de caracterización mencionados anteriormente, se desarrolló el tercer objetivo específico de la investigación el cual consistió en formular y aplicar una metodología que permitiera capacitar a los docentes de los programas de pregrado (profesional y tecnológico) en el enfoque cLIL con el fin de diseñar clases que integren el inglés a sus asignaturas. 
Una vez realizada una convocatoria interna para los docentes interesados, quienes respondieron fueron citados a una primera reunión informativa en donde se estableció un horario para encuentros semanales y se creó una base de datos compartida.

Para esta primera aproximación se contó con la inscripción de 37 docentes interesados, los cuales seleccionaron un horario de conveniencia para la capacitación, recibieron una presentación completa sobre el proyecto y la metodología de desarrollo. En la misma base de datos del archivo creado en Google Drive se incluyeron los temas que cada docente seleccionó para integrar el inglés en su clase y las asignaturas para la aplicación del diseño de actividades en el enfoque cLIL.

La capacitación consistió en recibir asesorías sobre la didáctica de la metodología que puede usar el docente para la enseñanza del tema escogido. Este proceso se llevó a cabo en un ambiente de desarrollo profesional en el que todos se consideran aprendices (Coyle, 20Io), bajo un trabajo colectivo y cercano entre colegas realizando observaciones de clases y haciendo un seguimiento reflexivo y crítico del proceso.

A través de los encuentros semanales, los docentes construyeron el diseño del planeador de clase CLIL (Bentley, 20Io) en el cual se aplicó cada uno de los aspectos del enfoque:

I. Las competencias a desarrollar tanto en el contenido de la asignatura como de la lengua extranjera inglés.

2. Los materiales físicos y virtuales que enriquecen el ambiente de aprendizaje.

3. El vocabulario, gramática y aspectos comunicativos específicos para el desarrollo de las competencias planteadas en el idioma.

4. La forma como se desarrollarían las 4C (principios de CLIL):

Contenido: La temática a desarrollar fue seleccionada por los docentes a partir de los contenidos programáticos de la asignatura. Teniendo en cuenta que el contenido implica el progreso en el nuevo conocimiento, habilidades y entendimiento (Coyle, Hood and Marsh, 20Io).

Cognición: Según las actividades que planearon los docentes identificaron y determinaron las habilidades cognitivas que orientan el proceso de aprendizaje, incorporando el pensamiento de orden superior, la solución de problemas de forma reflexiva para que el estudiante asuma los retos de la demanda cognitiva (Coyle, Hood and Marsh, 20Io). 
Estas deben conectarse con el contenido de manera creativa, su selección depende de la población a trabajar y de los objetivos de aprendizaje propuestos para cada actividad.

Comunicación. Aquí se unen el contenido y la demanda cognitiva con la comunicación, usando el lenguaje de aprendizaje (Language of learning) para explorar los nuevos conceptos, acceder al vocabulario y a la gramática acorde para la actividad, el lenguaje para el aprendizaje (Language for learning) para comunicar en lengua extranjera los conocimientos construidos a través de actividades productivas, y el lenguaje a través del aprendizaje (Language through learning) en donde se desarrolla el lenguaje vinculado al procesamiento cognitivo (Coyle, Hood and Marsh, 2010).

Cultura. Al tener en cuenta los aspectos socio-culturales se propende por el respeto por las diferencias, la calidad en las relaciones humanas que se pueden generar a través del trabajo colaborativo, el reconocimiento de las necesidades del entorno y el uso de herramientas de globalización, al estudiante ser consiente de sí mismo y del otro, de la identidad, la ciudadanía y el progreso a través del entendimiento pluricultural (Coyle, Hood and Marsh, 20I0). Krashen (I985, citado por Torga) explica ciertos fenómenos sobre los aprendizajes significativos desde la hipótesis del filtro afectivo y el entorno cultural: "El filtro afectivo es un bloqueo mental que evita que el estudiante utilice el input inteligible que recibe para la adquisición del lenguaje" (p.6). Una acción didáctica que tenga en cuenta el ambiente y la población con la que se trata disminuye los filtros afectivos para aprovechar los input inteligibles que permitan el aprendizaje de una lengua extranjera.

5. La organización de los tres momentos de la clase: antes, durante y después (University of Cambridge, 20I4), teniendo en cuenta el tiempo y la interacción de cada uno de ellos. En la enseñanza de una lengua extranjera es importante definir estos tres componentes en el planeador de clase para el desarrollo de las habilidades de Escucha, Lectura, Habla y Escritura con sus respectivas sub- habilidades (Spratt M, 2009): las habilidades Receptivas, Lectura y Escucha, son aquellas que no requieren de la creación del lenguaje, en las que el estudiante debe interpretar y negociar significados. Las habilidades Productivas implican Escritura y Habla las cuales implican la creación de lenguaje relacionado con una alta demanda cognitiva y el uso de estrategias interactivas. 
Los diseños de los planeadores de clase CLIL fueron vinculados por cada docente en su ejercicio pedagógico como parte de la internacionalización del currículo, política institucional de la ies. Esta formación docente comprendió un ciclo de dos semestres, uno para planear las actividades y otro para aplicarlas en clase. Durante el proyecto se realizaron 2 encuentros semanales por 32 semanas, en total se hicieron 64 encuentros para la preparación de los planeadores de clase, I9 docentes trabajaron en el primer ciclo y se completaron i I aplicaciones de actividades con enfoque cLIL.

Una vez los docentes ejecutaron el planeador de clases diseñado aplicando las actividades en el enfoque CLIL, y en desarrollo del cuarto objetivo de la investigación, se procedió a evaluar la efectividad del proyecto desde la perspectiva del docente y los estudiantes involucrados a través de una encuesta realizada a los participantes. A continuación, se presentan los resultados de las mismas.

Encuesta para docentes: la encuesta consta de un cuestionario escrito en inglés resuelto por 9 docentes quienes culminaron la última fase del proyecto: la aplicación del planeador de clase. Estos docentes participaron de los cursos de inglés de la siguiente forma: cinco docentes en nivel avanzado, dos docentes en nivel 2 y dos docentes en nivel introductorio, alcanzando así que las cuatro facultades de la institución y programas en extensión de la ies participaran en el proyecto.

Sin embargo, para el tratamiento de los resultados se tienen en cuenta las lecciones realizadas que en total fueron in, ya que un docente realizó tres actividades CLIL en tres grupos diferentes.

El primer segmento de la encuesta consta de 9 preguntas en las que el docente debía seleccionar la respuesta entre cuatro opciones a las que se les asignó un cuantificador: por debajo de las expectativas (I), justo lo necesario (2), bueno (3) y extraordinario (4) (véase figura 5).

Lo primero que se indagó fue si tras la experiencia vivida a través de la aplicación de la actividad cliL se considera que es posible enseñar contenidos a través del uso del inglés a lo cual el 80\% de los encuestados respondieron que es posible de forma extraordinaria y el $20 \%$ restante que lo es en buena medida. En cuanto a la posibilidad de aprender contenidos con el uso del idioma inglés, en el 90\% de las encuestas, los docentes respondieron que es posible de forma extraordinaria y el ıo\% de buena forma. 
Al indagar qué interés despertó el enfoque CLIL en los docentes, en el $70 \%$ de las aplicaciones la respuesta fue extraordinario y bueno para el 30\% restante. Otro de los aspectos importantes para medir la efectividad de la estrategia fue el indagar acerca de la mejora en la motivación de las clases al usar el inglés como medio de instrucción, el 60\% respondió que la motivación es extraordinaria, mientras para el 40\% respondió que es buena. Para el 70\% de las aplicaciones es extraordinaria la forma como la enseñanza integrando el inglés a los contenidos mejora el aprendizaje del inglés y viceversa.

Entre los docentes participantes, el $80 \%$ considera que es buena la forma en que se les facilita usar el inglés en sus clases, el 20\% restante de las aplicaciones lo considera extraordinario. El 80\% de las aplicaciones evidencia que el uso que hicieron los docentes de la metodología CLIL ayudó a mejorar su inglés en general de forma extraordinaria. Al comparar las clases CLIL con otras clases de inglés que han tenido, para el 70\% de los encuestados la calificación fue de extraordinaria, para el $20 \%$ buena y para el 10\% justo lo suficiente. Por tanto, al realizar una evaluación general de las actividades desarrolladas, para el 60\% de los docentes fue extraordinario y para el $40 \%$ fue buena.

Al aplicar el enfoque CLIL se esperaba que los docentes mejoraran sus competencias comunicativas en inglés, al consultarles al respecto se encontró que el proyecto contribuyó en un $65 \%$ a un nivel bueno y en un $27,5 \%$ a un nivel extraordinario como se muestra en la figura 6 .

El segundo segmento de la encuesta tiene como objetivo reconocer los aspectos positivos que los docentes evidenciaron al hacer uso del enfoque CLIL. Con el fin de definir de forma más precisa tales aspectos y aquellos por mejorar en la continuidad del proyecto, se realizaron tres preguntas abiertas dentro de las cuales se caracterizaron las respuestas.

(...) el maestro puede darse cuenta de la cantidad de actividades que pueden ejecutarse en el aula, las cuales aportaran no solo a un aprendizaje más productivo, sino también a la oportunidad de interiorizar los nuevos conocimientos de una manera más divertida y dinámica. En esta medida los estudiantes tendrán sus mentes abiertas lo cual les brinda la oportunidad de aprender más y mejor (García C. \& Gómez G., 2013, p.53). 
Figura 5. Resultados docentes

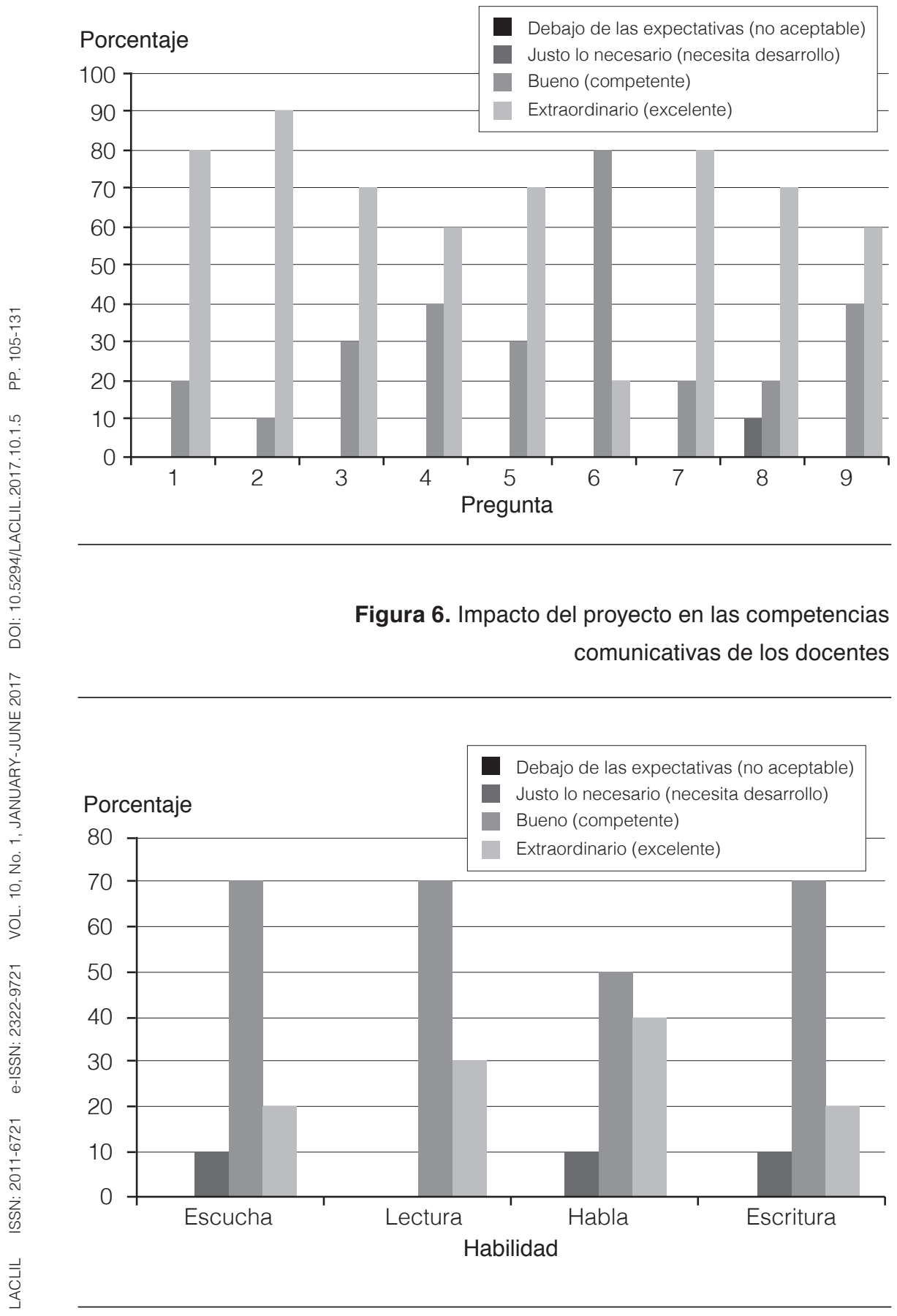


A continuación, se presentan los aspectos en orden decreciente de frecuencias en la contestación:

I. ¿Cuáles fueron los puntos más fuertes del proceso de aprendizaje usando CLIL?

" La motivación que generan los recursos didácticos usados y la novedad de la metodología a través del uso de textos interesantes y otras herramientas.

» El aprendizaje de los contenidos aun siendo presentados en otro idioma.

»El fortalecimiento en las habilidades de escritura, escucha y habla en inglés.

» La adquisición de vocabulario específico en inglés que permite expresar ideas y comunicarse sobre los temas de la profesión en esta lengua.

» La aplicación de lo aprendido de diversas formas y contextos.

2. ¿Tuvo alguna dificultad durante el proceso de aprendizaje usando CLIL?

» Los diferentes niveles de inglés en los que se encuentra un mismo grupo de estudiantes.

»El miedo causado por la inseguridad de no expresarse fluidamente en otro idioma.

» La carencia de los pre-saberes necesarios para comprender la lección.

» Falencias en el idioma inglés en relación con el vocabulario y la pronunciación.

»El tiempo que toma la preparación de las clases CLIL.

3. ¿Qué sugerencias tiene para mejorar el proceso de aprendizaje usando CLIL en el futuro?

»Aumentar las aplicaciones para un mismo grupo y expandirla a todas las asignaturas que se orientan.

» Continuar la capacitación en cLIL y en el idioma, y vincular otros docentes.

» Propiciar espacios y estímulos para que más docentes cátedra puedan vincularse. 
»Por último, se consultó a los docentes si recomiendan esta metodología a otros docentes a lo que el Io0\% afirmó positivamente.

Encuesta para estudiantes: la encuesta consta de un cuestionario escrito en inglés resuelto por II4 estudiantes con quienes se realizó la aplicación del planeador de clase CLIL. La información presentada a continuación corresponde a i a aplicaciones realizadas por los docentes. La participación comprendió una mixta población de estudiantes de primer a sexto semestre la mayoría cursando los niveles I, 2, 5 y 6 de inglés en una misma clase.

Al preguntar por la relevancia de usar el enfoque cLIL en la clase en la que se ejecutó el planeador de clase CLIL, para el 8I\% de los estudiantes fue bueno y tan solo para menos del $2 \%$ estuvo por debajo de las expectativas. Con el fin de ratificar la información anterior y para validar que el aprendizaje de los contenidos haya sido efectivo, se consultó a los estudiantes si consideran que es posible aprender contenidos a través del uso de inglés, y de acuerdo con los datos registrados, el balance es positivo ya que para ninguno de ellos está por debajo de las expectativas.

Uno de los objetivos del uso del enfoque CLIL en clase es la adquisición de vocabulario propio del área de estudio ya que a nivel profesional se hace necesario contar con el léxico específico que le permita hacer uso del inglés en un contexto profesional. Para el 72\% de los participantes, el aprendizaje de vocabulario fue bueno y para ninguno estuvo por debajo de las expectativas.

Una de las formas de medir el nivel de motivación que pudo generar el uso del enfoque cLIL cuando los estudiantes aprendieron y resolvieron problemas través del uso de otra lengua (Navés \& Muñoz, 2000), es indagar acerca de cuan interesante fue CLIL para ellos. Dentro de la encuesta, la respuesta de los participantes fue favorable en la medida en que sitúan la experiencia en un 50\% como bueno y en un 45,6\% como extraordinario.

Al aplicar el enfoque CLIL, se espera que los estudiantes mejoren sus competencias comunicativas en el inglés, al consultarles al respecto se encontró que para la mayoría el proyecto contribuyó en un nivel bueno en las habilidades de Escucha, Escritura, Lectura y Habla como se muestra en la figura 7 : 
Figura 7. Impacto del proyecto en la competencias comunicativas de los estudiantes

\section{Porcentaje}

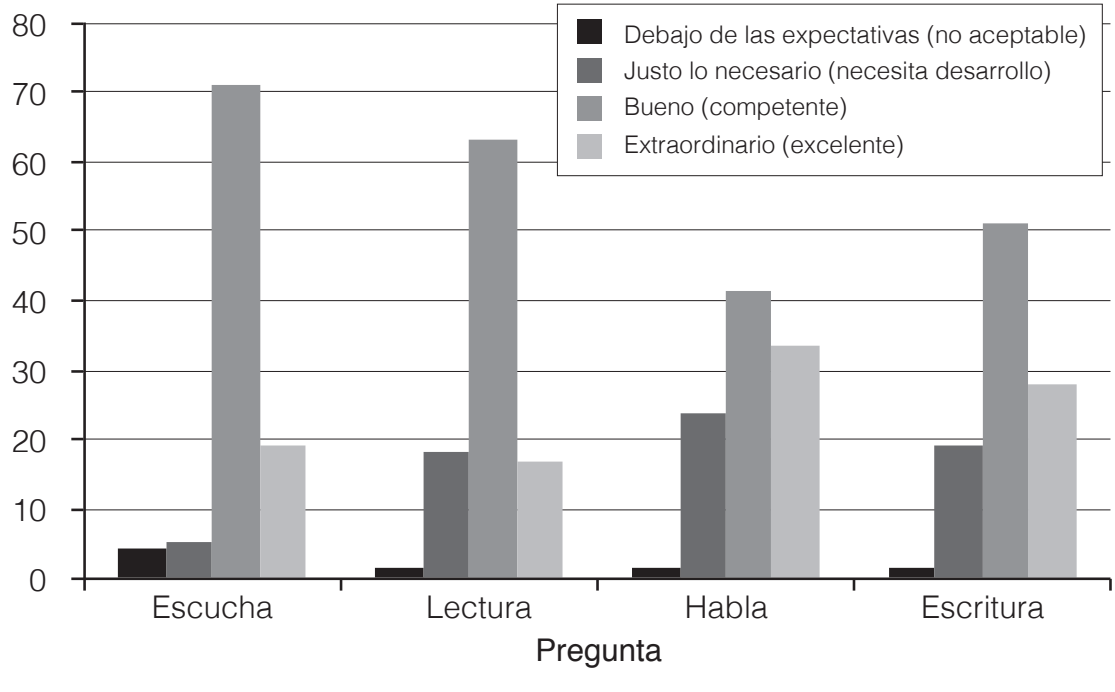

Al establecer una calificación general por parte de los estudiantes en cuanto a la metodología CLIL para el mejoramiento del inglés, los estudiantes determinaron en un $52 \%$ que fue bueno, un $36 \%$ extraordinario, un Io\% justo lo suficiente y tan solo para un ı\% no cumplió sus expectativas, lo cual representa un balance positivo para la investigación evidenciando el aprendizaje de contenidos y mejoras en las competencias comunicativas del inglés, tal como lo proponen otras investigaciones relacionadas con CLIL (Cantero, 20II).

Cuando los estudiantes involucrados en el proyecto comparan la clase en la que se ejecutó el planeador de clase clil con otras clases de inglés que han tenido, para el 57\% resulta buena la metodología usada, para ninguno estuvo por debajo de las expectativas.

Al indagar sobre la conveniencia del tiempo usado, para el $47 \%$ fue bueno y para el 37\% extraordinario, y tan solo para un 2,6\% estuvo por debajo de las expectativas. Al hacer una revisión general del proceso, los estudiantes lo evalúan en un 55\% como bueno y un $39 \%$ como extraordinario evidenciando una buena recepción por parte de los estudiantes. 
El segundo segmento de la encuesta tuvo como objetivo reconocer los aspectos positivos que los estudiantes evidenciaron en la ejecución de las actividades CLIL y para ello, se realizaron tres preguntas abiertas. En las respuestas de la primera pregunta se aprecia la coherencia entre características propias de este enfoque y su valoración por parte de ellos, en donde se destaca su apreciación acerca de la experiencia como una metodología nueva y muy interesante, y la importancia del docente comprometido. Las preguntas dos y tres fueron dirigidas a definir de forma más precisa, aspectos por mejorar para la posteridad del proyecto.

A continuación, se describen las respuestas en orden de frecuencia de menor a mayor:

I. ¿Cuáles fueron los puntos más fuertes del proceso de aprendizaje usando CLIL?

»Los contenidos específicos sobre el programa académico en la lección.

» El fortalecimiento de las habilidades en el inglés.

» La adquisición de vocabulario específico en inglés que permite expresar ideas y comunicarse sobre los temas de la profesión en esta lengua.

» La metodología fue nueva y muy interesante.

» La motivación para aprender inglés.

» La posibilidad de aprender contenidos específicos en otra lengua.

» Trabajo en equipo.

» La comunicación entre estudiantes y docentes.

» Refuerzo de los conocimientos en diferentes contextos.

» Tener conocimientos previos ayuda al desarrollo de la lección.

»Permite la autoevaluación de las competencias comunicativas del inglés.

» Contar con un buen docente.

» Hacer uso de textos interesantes y otros recursos.

2. ¿Tuvo alguna dificultad durante el proceso de aprendizaje usando CLIL?

»Carencias en las habilidades comunicativas del inglés.

»El contenido.

» Inexperiencia en esta metodología. 
»Poco tiempo.

» Faltó reforzar la pronunciación.

3. ¿Qué sugerencias tiene para mejorar el proceso de aprendizaje usando cLiL en el futuro?

»Aplicar con más frecuencia la metodología cLiL para la asignatura.

» Tener esta metodología en otras asignaturas.

» Aumentar el tiempo de la lección.

" Más lúdica.

» Aumentar la intensidad de aprendizaje del inglés.

» Hacer un glosario con las palabras nuevas en inglés.

Dada la variedad de asignaturas académicas en las que se impartieron las actividades CLIL, se contó con una población bastante mixta de estudiantes quienes tenían múltiples niveles de inglés, haciendo de la adaptación de los contenidos a través del uso de una segunda lengua, uno de los retos más grandes a enfrentar.

En promedio 7 de cada io estudiantes calificó como bueno las diferentes experiencias según los aspectos indagados y, por último, se les consultó si les gustaría tener otra clase utilizando la metodología CLIL, y si recomendaría el programa CLIL a otros estudiantes a lo cual el 97,4\% afirmo positivamente.

\section{Discusión}

La investigación aporta una metodología para la internacionalización del currículo que puede ser replicada en otras IEs, obteniendo como resultado principal la motivación positiva por parte de docentes y estudiantes. Debido a la naturaleza de la misma, entre los datos recolectados se encuentran las percepciones de los docentes y estudiantes frente al uso del enfoque cLIL en sus asignaturas, por esta razón se trata de un estudio preliminar que da pie a la realización de investigaciones posteriores en las cuales se realice una evaluación de la comprensión y aplicación de la metodología y de la mejora en las habilidades en la lengua extranjera Inglés de los maestros y estudiantes. 
Las encuestas diseñadas sirvieron como fundamento para la organización y generación de los cursos de inglés con el fin de fortalecer las competencias comunicativas en este idioma y como punto de partida para iniciar la propuesta de formación en el enfoque cLIL.

Es importante destacar la participación comprometida y la disponibilidad de tiempo con las actividades. Cada docente dedicó al menos 20 horas de trabajo cooperativo durante un semestre para lograr los objetivos de clase y se involucró en el proceso con miras a reforzar una carencia en la situación actual de la educación en la institución. Así mismo, desde la perspectiva y desarrollo del proyecto, todos los estudiantes superaron las expectativas de participación ya que el diseño de las lecciones se hicieron con el fin de fomentar el trabajo en equipo, mejorar la motivación e innovar en la dinámica y lúdica de las clases que comúnmente reciben (Caña de León, 2005).

En todas las preguntas formuladas en la encuesta para docentes, las respuestas fueron motivadoras, dejando en claro la gran relevancia de realizar proyectos internos y colaborativos entre los educadores de la institución. Más aún, dentro del grupo de trabajo, el idioma representaba para muchos una debilidad que llevó a formular si era completamente necesario tener una alta competencia en el idioma inglés, sin embargo, se denota en los resultados finales alcanzados, que a pesar de no poseer un alto conocimiento en esta lengua extranjera, el acompañamiento del docente asesor cliL (quien realizó visitas a las clases de los docentes), la preparación y adquisición de los conocimientos previos y las herramientas necesarias, permiten llevar a cabo las actividades sin mayores contratiempos.

Los docentes involucrados no solo evidenciaron las muchas posibilidades de usar el enfoque CLIL, sino que también entendieron cómo funciona y que aspectos se deben tener en cuenta para su uso. El proceso de formación en este enfoque requiere de varias etapas en las que es necesario consolidar los aspectos teóricos, la metodología, el desarrollo de actividades y evaluación de resultados. Para la construcción del planeador de clases CLIL el docente debió reflexionar acerca del contenido que consideraba podía enseñar a través del idioma inglés y las competencias que esperaba que los estudiantes desarrollaran en el área de conocimiento. Con la asesoría de un docente experto en la metodología y en el idioma inglés se determinaron las competencias 
propias del idioma necesarias para cada grupo específico de estudiantes, teniendo en cuenta el nivel promedio de inglés que presentaban.

Los nueve profesores que realizaron las actividades con enfoque CLIL, cambiaron su metodología de enseñanza y modificaron las actividades que realizaban comúnmente para exponer el contenido a sus estudiantes. En promedio 6 de los 9 docentes participantes consideró como extraordinaria la experiencia en todos los aspectos indagados, y para todos quedó claro que es necesario involucrar a más educadores en el proceso que, en consecuencia, con los resultados positivos obtenidos, llevarán a una segunda fase este proyecto de investigación.

Dentro del análisis general de la información colectada a través de las encuestas a los estudiantes, un I2\% aproximadamente del total de la población universitaria hizo parte fundamental en la determinación de la efectividad del proyecto el cual se consideró efectivo desde la perspectiva de sus participantes.

En concordancia con los objetivos planteados, el 90\% de los docentes y el $77 \%$ de los estudiantes opinaron de forma altamente positiva que el aprendizaje de contenidos es posible a través del inglés y que a su vez se adquiere también más conocimiento fortaleciendo las competencias comunicativas en el uso de esta lengua extranjera a través de las aplicaciones de clases con enfoque ciriL.

Uno de los aspectos más importantes que comparten en opinión los docentes y estudiantes del proyecto, es en el que se exalta la necesidad prioritaria del aprendizaje del inglés, todos concuerdan en la relevancia de tener las competencias en el idioma tanto para enseñar como para aprender. Al comparar las opiniones acerca de las dificultades que presentaron ambos, docentes y estudiantes, durante el desarrollo de las actividades CLIL, se determinó que la diferencia entre los niveles de inglés, el cual no correspondía al requerido en el semestre que cursaban los estudiantes, representó un desafío para la aplicación de las actividades.

Otro aspecto relevante que compartieron docentes y estudiantes dentro del proceso de aprendizaje usando cliL, fue el uso innovador de los contenidos, la comunicación con el estudiante, la autoevaluación del inglés y el trabajo en equipo el cual incentiva el aprendizaje colaborativo ya que "el aprendizaje se realiza a partir de la cooperación de todos los miembros del grupo, esta cooperación para la construcción 
del conocimiento amplía las necesidades comunicativas, un aspecto fundamental para el desarrollo de la competencia comunicativa" (Cerdá Vallés, 20I4).

Por su origen innovador para la iEs, la metodología cLIL se debe desarrollar con tiempo y creatividad, la preparación de las actividades se realizó de manera colectiva con el fin de integrar y mejorar la relación que la población universitaria tiene en general con el idioma inglés. Estudiantes y maestros lograron ver a través de este proyecto la congruencia de una puesta en común, en donde se destaca el rol del docente y se tiene como eje principal las habilidades de los estudiantes.

\section{Referencias}

Bentley, K. (2010). The TKT Course cLIL Module. Cambridge University Press. Cantero García, V. (20II). La enseñanza de segundas lenguas a través de tareas: una propuesta didáctica para I de Eso biblingüe. Dialnet(I7), I33-I56.

Caña de León, P. (2005). La Innovación Educativa. Madrid: Akal S.A.

Cerdá Vallés, C. \&. (20I4). El Aprendizaje Cooperativo para el desarrollo de la Competencia Comunicativa Oral en Lengua Inglesa en el aula de primaria. Encuentro. Revista de investigación e innovación en la clase de idiomas, (23), I6-29.

Chomsky, N. (1965). Aspects of the Theory of Syntax. Cambridge: MiT Press.

Coyle, D. H. (20IO). The CLIL Tool Kit: Transforming theory into practice. CLIL: Content and Language Integrated Learning. Cambridge.

García C., L. C., \& Gómez G., M. d. (2013). Efectos del uso del CLIL aplicado a ciencias naturales en el desempeño en inglés de los estudiantes de grado segundo.Tesis de grado presentada como requisito para obtener el título de Licenciada en Básica primaria con énfasis en inglés. Manizales: Universidad de Manizales.

Ministerio de Educación Nacional. (20I4). Ministerio de Educación Nacional . Retrieved from Programa Nacional de Inglés "Colombia very well": www.mineducacion.gov.co 
Navés, T., \& Muñoz, C. (2000). Usar las lenguas extranjeras para aprender y aprender a usar las lenguas extranjeras.Una introducción a AICLE para madres, padres y jóvenes. In Marsh, D., \& Langé, G. (eds.), Using Languages to Learn and Learning to Use Languages. Jyväskylá: Finland: UniCOM, University of Jyväskylä on behalf of TIE-CLIL.

Sabino, C. (1992). El Proceso de Investigación. Caracas: Ariel.

Spratt, M. (2009). Module I: Describing language skills and subskills - Teacher's. In P. A. Spratt M, The TKT Course (p. 3). University of Cambridge ESOL Examinations.

Suárez, M. L. (2005). Claves del éxito del aprendizaje integrado de contenidos y lengua extranjera (AICLE). Obtenido de Charla presentada en la 5ta Jornada sobre Aprendizaje Cooperativo del grupo GIAC. Bilbao: Universidad de Deusto. http://giac.upc.es/pag/giac_cas/giac_jac/o5/ jaco5-mls.htm

Torga, M. (20I0). Vigotsky y Krashen: Zona del desarrollo próximo y el aprendizaje de una lengua extranjera. Universidad Nacional de Comahue.

Unesco. (20II). Unesco. Retrieved from Unesco and Education: "Everyone has the right education": www.unesco.org

University of Cambridge. (20I4). Cambridge English Teaching Framework. Cambridge English Language Assessment. 


\section{Apéndice 1}

Tabla 2. Preguntas de la prueba de caracterización.

\begin{tabular}{|c|c|}
\hline & Pregunta \\
\hline 1 & $\begin{array}{l}\text { ¿Qué grado de importancia tiene el dominio de un segundo idioma en } \\
\text { su formación profesional? }\end{array}$ \\
\hline 2 & $\begin{array}{l}\text { ¿Qué grado de importancia tiene el dominio de un segundo idioma en } \\
\text { su vida profesional? }\end{array}$ \\
\hline 3 & $\begin{array}{l}\text { ¿En qué medida opina que se puede relacionar la enseñanza del } \\
\text { idioma extranjero con la de otras asignaturas? }\end{array}$ \\
\hline 4 & $\begin{array}{l}\text { ¿Opina que se puede adaptar la enseñanza actual de otras asignaturas } \\
\text { usando el inglés? }\end{array}$ \\
\hline 5 & $\begin{array}{l}\text { ¿Piensa que está preparado y capacitado para orientar sus asignaturas } \\
\text { usando el inglés? }\end{array}$ \\
\hline 6 & $\begin{array}{l}\text { ¿Piensa que mejoraría la motivación de sus clases el enseñar utilizando } \\
\text { el inglés? }\end{array}$ \\
\hline 7 & $\begin{array}{l}\text { ¿Cree usted que mejoraría el aprendizaje de sus alumnos en el idioma } \\
\text { extranjero si se trabaja a través del inglés en sus asignaturas, y } \\
\text { viceversa? }\end{array}$ \\
\hline 8 & $\begin{array}{l}\text { ¿Considera usted que adquiriría un mejor dominio del idioma extranjero } \\
\text { si enseñara usando el inglés en sus asignaturas? }\end{array}$ \\
\hline 9 & $\begin{array}{l}\text { ¿Considera que enseñar asignaturas diferentes del inglés en este } \\
\text { idioma sería sencillo para usted? }\end{array}$ \\
\hline
\end{tabular}

